\title{
Evaluation of Oxidative Stress, Antioxidant Power, and Antioxidant Potential of Breastmilk of Breast-Feeding Mothers
}

\author{
Naoko Kuramoto $^{1,2^{*}}$, Mariko Kitagawa ${ }^{3}$ \\ ${ }^{1}$ Department of Reproductive Health Nursing, School of Nursing, University of Human Environments, Obu, Japan \\ ${ }^{2}$ Department of Reproductive Health Nursing, Graduate School of Nursing, Nagoya City University, Nagoya, Japan \\ ${ }^{3}$ Department of Reproductive Health Nursing, Graduate School of Nursing, University of Human Environments, Obu, Japan \\ Email: *n-kuramoto@uhe.ac.jp
}

How to cite this paper: Kuramoto, N. and Kitagawa, M. (2017) Evaluation of Oxidative Stress, Antioxidant Power, and Antioxidant Potential of Breastmilk of BreastFeeding Mothers. Health, 9, 1145-1158. https://doi.org/10.4236/health.2017.98083

Received: July 5, 2017

Accepted: August 18, 2017

Published: August 21, 2017

Copyright @ 2017 by authors and Scientific Research Publishing Inc. This work is licensed under the Creative Commons Attribution International License (CC BY 4.0).

http://creativecommons.org/licenses/by/4.0/ (c) (i) Open Access

\begin{abstract}
Purpose: The purpose of this study was to evaluate oxidative stress, antioxidant power, and antioxidant potential of breastmilk of breast-feeding mothers from the early postpartum period to the first 3 months postpartum, and to examine the dynamics and the relationships among them. Method: For 47 puerperant women who gave vaginal birth without any pregnancy complications, the oxidative stress levels (d-ROMs levels) and antioxidant power (BAP levels) in the maternal plasma as well as antioxidant potential of breastmilk (BAP levels in breastmilk) were measured 3 times, i.e., in the early puerperium (4 or 5 days after giving birth), one month after giving birth, and 3 months after giving birth. Results: The d-ROMs levels in the maternal plasma were significantly high in the early puerperium $(p<0.001)$, and decreased gradually in the postpartum period $(p<0.001)$. On the other hand, BAP levels were significantly low in the early puerperium $(p<0.001)$, and increased to the almost normal level during one month after giving birth. BAP levels in breastmilk were significantly high in the early puerperium compared with the other periods $(p<0.001)$, and decreased gradually until 3 months after giving birth $(p<0.001)$. BAP levels in breastmilk in the early puerperium were higher compared with the maternal BAP levels, and there was a positive correlation between BAP levels in breastmilk and those in the maternal plasma $(p<$ $0.05)$. Discussion: Regarding the oxidative stress and the antioxidant defense system of breast-feeding mothers, the d-ROMs level was highest and the BAP level was lowest in the early puerperium. The BAP level then showed a clear tendency to recover in the first 3 months after giving birth. Conclusion: When the maternal antioxidant potential remains at a low level after giving birth, careful consideration should be given to the mother's health and wellbeing
\end{abstract}


because there is a possibility that it might affect the antioxidant potential of breastmilk.

\section{Keywords}

Oxidative Stress, Antioxidant Power, Maternal Plasma, Breastmilk, Postpartum

\section{Introduction}

Oxidative stress occurs when there is a loss of balance between the production of the reactive species (RS) in the living organisms and the antioxidant power regulated by both antioxidant enzymes and the antioxidants involved in the living organisms and when consequently oxidation becomes so predominant that it causes oxidative damage to the living organisms [1]. Pregnancy is believed to be the period when oxidative stress is enhanced by increased mitochondrial activity due to active energy metabolism in the placenta as well as by increased production of active oxygen species [2].

Research shows that oxidative stress plays a role in the onset of pregnancy complications; it is deeply associated with the pathogenic mechanism of pregnancy complications. Pregnancy-induced hypertension syndrome is one of the especially important pregnancy complications, and it is reported that damage of vascular endothelium due to the enhanced oxidative stress could be a factor of onset or progression of the disease [2] [3] [4]. An animal study reported that complications of diabetes mellitus during pregnancy are associated with incidence of fetal malformation due to hyperglycemia and with increased production of active oxygen [5], and clinical studies are ongoing to clarify the underlying cause and to prevent the disease [6] [7]. It is also considered that the difference of mode of delivery may influence the oxidative stress. In fact, a study reported that 8 -OHdG levels in the prolonged delivery group were higher than those in the natural childbirth group [8]. Moreover, it was pointed out that the increase of oxidative stress in the delivery period was influenced by exertion of skeletal muscles and uterine muscles over a long period of time. Furthermore, there is a report that 8-isoprostane and 8-OHdG, i.e. makers of oxidative stress, increased in the placental tissues in the vaginal birth group compared with the selective cesarean delivery group and that $8-\mathrm{OHdG}$ and antioxidant potential in the umbilical cord blood increased in the vaginal birth group [9]. The increase of oxidative stress was found not only in a mother's body but also in the fetoplacental system.

These findings suggest that labor pain is associated with the enhancement of oxidative stress in the placenta. It was also reported that an increase of malondialdehyde (MDA; a metabolite of lipoperoxidation) and superoxide dismutase (SOD; an antioxidant enzyme) was observed in the umbilical cord blood of babies who presented a non-reassuring fetal status pattern on the intrapartum fetal 
cardiotocogram [10]. Currently, use of oxidative stress marker as an evaluation index of fetal dysfunction and acute/chronic hypoxia is being tested.

Although previous studies on perinatal oxidative stress mainly dealt with elucidation of pathology and prevention of pregnancy complications such as pregnancy-induced hypertension syndrome and diabetes, recent studies have been focusing even on collecting data in the delivery period. However, few studies have examined oxidative stress especially in the puerperium. In recent years, it has been pointed out that autoimmune disorders such as chronic rheumatoid arthritis and SLE occur highly frequently in the puerperium [11] and an increase of thioredoxin (TRX), a biomarker, has been confirmed in the puerperium, suggesting an association between TRX and inflammatory cells [12]. This result indicates that oxidative stress is involved in the exacerbation of autoimmune disease in the puerperium. However, it remains to be seen as to what exact changes caused by the oxidative stress during pregnancy will surface after delivery, i.e., in the puerperal period. Moreover, few studies have evaluated antioxidant potential of breastmilk by looking at the correlation between maternal oxidative stress and antioxidant power. Furthermore, our findings show that no longitudinal followup study was ever conducted.

Based on the above observations, the purpose of this study was set to clarify the dynamics of oxidative stress, antioxidant power, and antioxidant potential of breastmilk of breast-feeding mothers up to 3 months after giving birth. In this study, we discuss the relationship between the status of maternal oxidative stress and antioxidant power as well as antioxidant potential of breastmilk. We hope that findings on the oxidative stress of breast-feeding mothers and on the role of antioxidant defense system will help make the oxidative stress markers available an objective indicator to assess the perinatal period and manage women's health.

\section{Materials and Methods}

\subsection{Participants}

This study was conducted at maternity hospitals in the Chubu region in Japan. The participants were puerperants who had a natural childbirth, and their data were collected in the period from June 2012 to April 2013. Inclusion criteria were as follows: puerperants who 1) had no pregnancy complications and had a normal pregnancy, 2) had no specific medical history, 3) had a singleton pregnancy, and 4) agreed to participate in the study. When we calculated the number of samples that were necessary to conduct ANOVA using a repeated-measure design among the three groups, with the $\alpha$-level, the power, and the medium effect size defined as $0.05,0.08$, and 0.25 , respectively, we identified 29 samples. Therefore, sampling was performed aiming to obtain 50 samples. Out of the 55 participants who were asked to cooperate in the research, 47 participants who could be tracked for up to 3 months after giving birth were analyzed in the study.

This study was approved by the Ethical Committee of Nagoya City University 
School of Nursing. The aim of this study was explained orally and in a written format, and written informed consent was obtained from all participants.

\subsection{Analytical Methods}

In this study, both blood and breastmilk were collected from the participants to analyze oxidative stress level, antioxidant power, and antioxidant potential of breastmilk. Blood and breastmilk were collected 3 times: in the early puerperium ( 4 or 5 days postpartum), one month after giving birth, and 3 months after giving birth. About $1 \mathrm{ml}$ of blood sample was collected from the vein in the inner elbow into a microcuvette containing heparin. Each resultant blood sample was centrifuged to isolate plasma components at $6000 \mathrm{rpm}$ for $2 \mathrm{~min}$. About $1-5 \mathrm{ml}$ of breastmilk was manually expressed into a sterile tube. The collected breastmilk was centrifuged into creamy component and defatted milk, and the defatted milk was collected. The collected samples were analyzed by using F.R.E.E. (Free Radical Elective Evaluator; Diacron, Italy) within 72 hours. Specifically, oxidative stress levels and antioxidant power of maternal plasma were analyzed by measuring derivatives of reactive oxygen metabolites (d-ROMs) levels and biological antioxidant potential (BAP) levels, respectively, while antioxidant potential of breastmilk was analyzed by measuring BAP levels.

Basic information regarding each individual's characteristics was collected via their medical records and birth records. Specifically, the following information was collected: age, medical history, pregnancy/childbirth history, height/weight during non-pregnancy, abnormality during the course of pregnancy, pregnancy anemia, the number of weeks at delivery, abnormal course of delivery, duration of labor, the amount of bleeding during delivery, abnormal puerperal period, problems with nursing methods/period, and postpartum weight. In addition, BMI (body mass index) was calculated by using height and weight at the following each time point: during non-pregnancy, in the early puerperium, one month after giving birth, and 3 months after giving birth.

1) Derivatives of reactive oxygen metabolites (d-ROMs)

Carratelli et al. in Italy developed a d-ROMs test for the purpose of performing a comprehensive evaluation of oxidative stress [13] [14]. This test has enabled to evaluate oxidative stress easily in a short period of time with high reproducibility, and has helped to conduct advanced clinical studies. The d-ROMs test is a test to measure the blood concentration of hydroperoxide, a metabolite of active oxygen and/or free radicals, by color reaction and to evaluate the in vivo status of oxidative stress levels comprehensively. During the d-ROMs test, hydroperoxide in a blood sample produces alkoxyl and peroxyl radicals via the Fenton reaction in the presence of iron which is released from plasma protein by the acidic buffer (the $2^{\text {nd }}$ reagent $\mathrm{R}-2$ in the attached kit). These radicals gradually oxidize colorless alkyl-substituted aromatic amines that are dissolved in the dye mixture (the $1^{\text {st }}$ reagent $\mathrm{R}-1$ in the attached kit) one after another and produce pink-colored derivatives. This pink-colored reaction was measured by using 
a photometer ( $505 \mathrm{~nm} ; 5 \mathrm{~min}$ ). The result of the d-ROMs test was expressed in Carratelli units (U.CARR). 1 U.CARR represents $0.08 \mathrm{mg} / \mathrm{dl}$ of hydrogen peroxide water.

2) Biological antioxidant potential (BAP)

Antioxidant power of maternal plasma and antioxidant potential of breastmilk were determined by using a BAP test [15]. This BAP test is a test to measure the ability of reduction substance in the samples, that is, the power to reduce trivalent iron $\left(\mathrm{Fe}^{3+}\right)$ into bivalent iron $\left(\mathrm{Fe}^{2+}\right)$, in order to evaluate antioxidant power. BAP test is a test that utilizes the same chemistry principle as Ferric Reducing Antioxidant Power (FRAP) assay to measure antioxidant power of biological fluid samples and foods. $50 \mu \mathrm{l}$ of trivalent iron salt $\left(\mathrm{FeCl}_{3}\right.$ : the $2^{\text {nd }}$ reagent $\mathrm{R}-2$ in the attached kit) was added to a cuvette containing thiocyanate derivative solution (the $1^{\text {st }}$ reagent $\mathrm{R}-1$ in the attached kit). The reagent reddened by trivalent iron ions $\left(\mathrm{Fe}^{3+}\right)$ was measured by using a photometer. Then, $10 \mu \mathrm{l}$ of plasma or defatted milk was added into the cuvette and the changes were measured again using a photometer $(505 \mathrm{~nm})$ for 5 minutes, during which the trivalent irons were reduced to bivalent irons $\left(\mathrm{Fe}^{2+}\right)$ and decolorized by plasma antioxidants. The unit was expressed as either $\mu \mathrm{mol} / \mathrm{L}$ or $\mu \mathrm{Eq} / \mathrm{L}$.

\subsection{Statistical Analysis}

IBM SPSS Statistics 19.0 was used for all the statistical analyses. A one-factor repeated measure analysis of variance (ANOVA) was performed to evaluate the changes caused by d-ROMs levels and BAP levels in the maternal plasma as well as breastmilk BAP levels during the puerperal period, followed by Bonferroni's multiple comparison test. The mean and the standard deviation were used to describe each measurement value. Pearson's correlation analysis was used to investigate the relationship among d-ROMs levels, BAP levels, and breastmilk BAP levels. $P$ value of $<0.05$ was defined as statistically significant in all the analyses.

\section{Results}

\subsection{Characteristic of Participants}

Table 1 shows the participants' characteristics. The mean age was $32.7 \pm 4.5$ years (range: 20 to 41 years). Primiparas and multiparas accounted for $17.0 \%$ and $83.0 \%$ of the study population, respectively. Of the multiparas, biparas and triparas accounted for $59.0 \%$ and $41.0 \%$, respectively. $78.7 \%$ of pregnant women were found to have anemia. No one developed pregnancy complications such as preeclampsia. All the women had a normal delivery, with the mean gestational age at delivery being $39.1 \pm 1.1$ weeks (range: 36 to 42 weeks). Neonatal weight was $3194.2 \pm 305.2 \mathrm{~g}$ (range: 2668 to $4070 \mathrm{~g}$ ). The amount of bleeding during delivery was $323.7 \pm 207.8 \mathrm{~g}$ (range: 90 to $908 \mathrm{~g}$ ).

The mean duration of labor was $6.5 \pm 5.3$ hours (range: 1.1 to $31.2 \mathrm{hrs}$.). Compared between primiparas and multiparas, the mean duration of labor of primiparas was significantly longer than that of multiparas (primiparas: $13.7 \pm$ 
Table 1. Participants characteristics.

\begin{tabular}{cc}
\hline Age (years old) & $N=47$ \\
\hline Gestational week (weeks) & $32.7 \pm 4.5$ \\
Non-pregnant BMI & $39.1 \pm 1.1$ \\
Body weight gain during pregnancy (Kg) & $20.8 \pm 3.3$ \\
BMI 1 month after giving birth & $9.9 \pm 3.7$ \\
BMI 3 months after giving birth & $22.1 \pm 3.0$ \\
Number of delivery primipara & $21.6 \pm 3.0$ \\
Multipara & $8(17.0 \%)$ \\
Experience of pregnancy anemia & $39(83.0 \%)$ \\
Amount of intrapartum hemorrhage (ml) & $37(78.7 \%)$ \\
Whole delivery time (hours) All & $323.7 \pm 207.8$ \\
Primipara & $6.5 \pm 5.3$ \\
Multipara & $13.7 \pm 9.2$ \\
Birthweight of a newborn baby (g) & $5.0 \pm 2.2$ \\
\hline
\end{tabular}

Data are presented by mean \pm SD or number (\%).

Table 2. Changes of maternal oxidative stress (d-ROMs levels) and antioxidant power (BAP levels), and antioxidant potential of breastmilk (Breastmilk BAP levels) from the early postpartum period to the first 3 months postpartum $(N=47)$.

\begin{tabular}{cccccc}
\hline & $\begin{array}{c}\text { Early postpartum } \\
\text { period } 4.6 \pm 0.5 \\
\text { days }\end{array}$ & $\begin{array}{c}1 \text { month after } \\
\text { giving birth } 36.4 \pm \\
4.4 \text { days }\end{array}$ & $\begin{array}{c}3 \text { months after } \\
\text { giving birth } 98.5 \pm \\
4.5 \text { days }\end{array}$ & $F$ value & $P$ \\
\hline $\begin{array}{c}\text { d-ROMs levels } \\
(\mathrm{U} . \mathrm{CARR})\end{array}$ & $662.9 \pm 105.6^{\mathrm{a}, \mathrm{b}}$ & $414.0 \pm 69.9^{\mathrm{b}}$ & $371.6 \pm 51.3$ & 333.38 & 0.000 \\
$\begin{array}{c}\text { BAP levels } \\
(\mu \mathrm{mol} / \mathrm{L})\end{array}$ & $1608.9 \pm 282.0^{\mathrm{a}, \mathrm{b}}$ & $2055.5 \pm 263.4$ & $2089.6 \pm 156.2$ & 97.62 & 0.000 \\
$\begin{array}{c}\text { Breastmilk BAP } \\
\text { levels }(\mu \mathrm{mol} / \mathrm{L})\end{array}$ & $4333.6 \pm 603.0^{\mathrm{a}, \mathrm{b}}$ & $3824.4 \pm 443.8^{\mathrm{b}}$ & $3127.8 \pm 244.5$ & 113.08 & 0.000 \\
\hline
\end{tabular}

Data are presented by mean $\pm \mathrm{SD},{ }^{\mathrm{a}} p<0.001$ versus 1 month after giving birth; ${ }^{\mathrm{b}} p<0.001$ versus 3 months after giving birth.

9.2 hours; multiparas: $5.0 \pm 2.2$ hours) [ $t(45)=2.64, p=0.033$ ]. There was no significant difference between primipara and multipara women regarding the other attributes except the duration of labor, such as age, the number of weeks at delivery, BMI, the presence of pregnancy anemia, the amount of bleeding during delivery, and the birthweight of an infant.

\subsection{Changes of Oxidative Stress Levels, Antioxidant Power, Antioxidant Potential of Breastmilk of Breastfeeding Mothers}

Table 2 shows the average, the standard deviation, and the results of the analysis of variance of the maternal d-ROMs levels, BAP levels, and BAP levels in breastmilk in the early puerperium, one month after giving birth, and 3 months 
after giving birth. The d-ROMs level was high in the early puerperium, but decreased significantly in the first month and 3 months after giving birth. Multiple comparison revealed a significant difference in all the periods $(p<0.001)$. On the other hand, the BAP level increased significantly during from the early puerperium up to one month after giving birth, and remained unchanged up to 3 months after giving birth. Multiple comparison revealed a significant difference between the early puerperium and one month after giving birth and between the early puerperium and 3 months after giving birth $(p<0.001)$. The BAP level in breastmilk was highest in the early puerperium and decreased significantly as time progressed until 3 months after giving birth. Multiple comparison revealed a significant difference between all the periods $(p<0.001)$.

\subsection{Relationship among Oxidative Stress Levels, Antioxidant Power, and Antioxidant Power of Breastmilk of Breastfeeding Mothers}

Compared among the three periods, i.e., in the early puerperium $(r=-0.081$, n.s.), one month after giving birth $(r=-0.034$, n.s. $)$, and 3 months after giving birth $(r=0.084, n . s$.$) , there was no correlation between the maternal d-ROMs$ levels and the BAP levels in breastmilk. Figure 1 shows the correlation between the maternal BAP levels and the BAP levels in breastmilk in those three periods. A significant correlation was observed between the maternal BAP levels and the BAP levels in breastmilk in the early puerperium $(r=0.335, p<0.05)$.

\section{Discussion}

In this study, we measured the maternal oxidative stress levels from the d-ROMs levels and the antioxidant power from the BAP levels in the three periods, i.e., in the early puerperium, one month after giving birth, and 3 months after giving birth in order to evaluate the antioxidative defense mechanism in the lactation period. We also evaluated the antioxidant potential of breastmilk using the BAP levels in breastmilk. The maternal oxidative stress levels (d-ROM levels) decreased
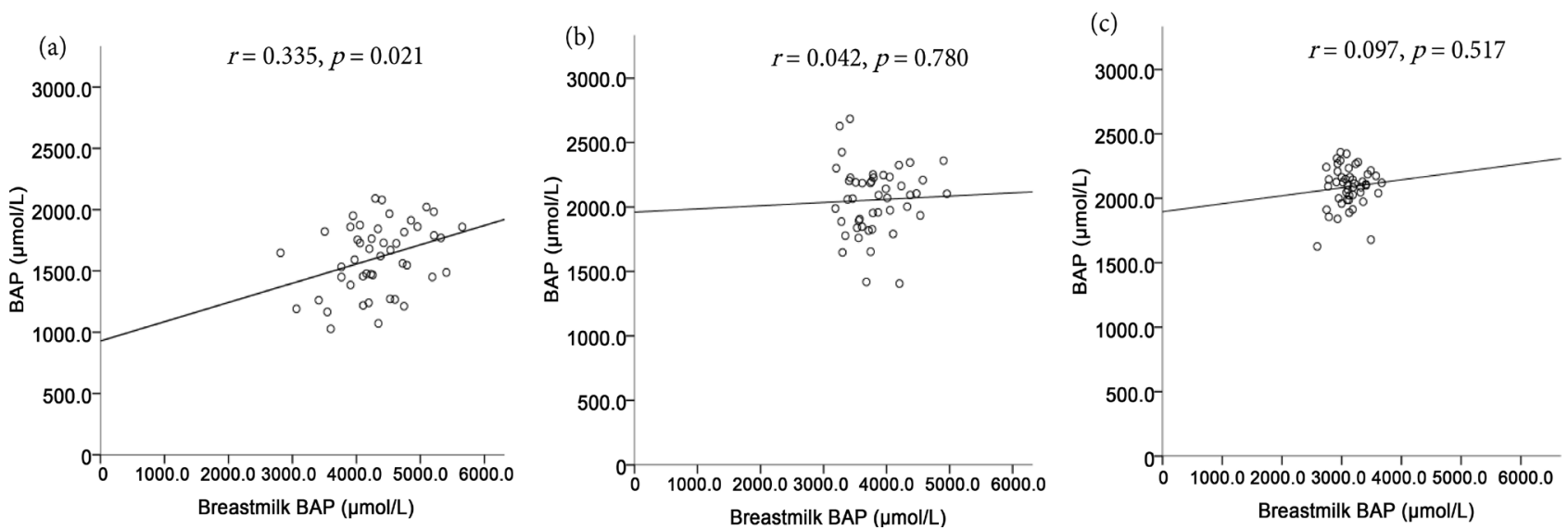

Figure 1. Correlation between antioxidant power (BAP levels) of maternal plasma and antioxidant potential of breastmilk (Breastmilk BAP levels) $(N=47)$ : early postpartum period (a), 1 month after giving birth (b), 3 months after giving birth (c). 
gradually as the puerperal period progressed, but it was $371.6 \pm 51.3$ U.CARR even 3 months after giving birth, which was higher than the standard value (200 - 300 U.CARR).

The d-ROMs level in pregnant women is reported to increase at the end of pregnancy even if they don't have any complications, compared with the nonpregnancy period [16] [17] [18], while the d-ROMs level during delivery is pointed out to become higher than 600 U.CARR [19]. A previous study [20] had already described that the d-ROMs levels were higher during the first 0 - 2 postpartum days, but it was found out that they were still high at 3 months after giving birth.

It was also revealed that the oxidative stress level would need some time to return to the normal. Based on the viewpoint of women's lifelong health management, we think that we need to follow changes over the time to better understand when the oxidative stress in the perinatal period would return to normal. Moreover, when a high level of oxidative stress continues, we need to encourage those women to have a consultation considering potential influence of other diseases. Furthermore, it has been made clear that oxidative stress levels of puerperants who have developed complications such as pregnancy induced hypertension syndrome during pregnancy will increase as compared with women with normal pregnancy [2] [3]. In addition, a significant increase in the indexes of lipoperoxidation and oxidative stress in the maternal blood and the umbilical cord blood before the onset of labor as well as a significant decrease of the antioxidant potential was reportedly observed more in pregnant women who got complicated with hypertension such as preeclampsia and pregnancy-induced hypertension syndrome than in normal pregnant women [21]. Another study reported that there was a positive correlation between the carbonyl group (i.e., an oxidative stress marker) and lipoperoxidation between the maternal blood and the umbilical cord blood during childbirth [22]. These findings indicate that the oxidative stress status of mothers and newborns are mutually correlated.

The d-ROMs level in the umbilical cord blood of women with preeclampsia who got complicated with intrauterine growth restriction (IUGR) was also reportedly significantly higher than those who were not complicated with IUGR [16]. Women with pregnancy complications are likely to experience an increase of oxidative stress, and the high oxidative stress level can be transmitted to newborns through the umbilical cord blood. Therefore, attention must be paid to the potential high level of oxidative stress a newborn has when the maternal oxidative stress is continuously at high level since pregnancy.

On the other hand, the maternal antioxidant power increased close to the standard value ( $\geq 2200 \mu \mathrm{mol} / \mathrm{L})$ at one month after giving birth. Given that the antioxidant power at one month after giving birth was almost same as that at 3 months after giving birth, it was understood that the antioxidant power would recover one month after giving birth. In a previous study [20], the antioxidant power at one month after giving birth increased to the standard value. In our 
study, however, the antioxidant power at one month and 3 months after giving birth were lower than that. Given that the antioxidant power will recover at one month after giving birth, the puerperants in our study might belong to a population where the value of the pre-pregnancy baseline was low compared to previous studies. This population is probably the one wherein the baseline value of the antioxidant power is low in usual conditions, i.e., during non-pregnancy.

Health is maintained by a fine, in-vivo balance between the production of active oxygen and the antioxidative defense mechanism. Ideally, women planning to become pregnant should receive a premarital health screening to evaluate whether they are able to become pregnant or they can lead a healthy pregnant life. Evaluating the oxidative stress as one of the items of health screening enables to grasp the changes of the oxidative stress from the baseline in the 3 periods, i.e., during pregnancy, in the delivery period, and in the puerperium. Admittedly, comparison of the oxidative stress with the normal range is also important. If the individual's baseline value is obtainable, evaluating the amount of relative change from the baseline value will also enable to evaluate the individual's oxidative stress status more appropriately. It is quite rightful to suspect the effect of oxidative stress when a sudden change of oxidative stress markers from the baseline value was observed. It is important to analyze the oxidative stress status comprehensively in terms of both oxidative stress and antioxidant power in order to evaluate the in vivo oxidative stress status. Admitting that the antioxidant power will improve one month after giving birth, puerperants with low antioxidant power can be considered to have relatively high oxidative stress, because the oxidative stress levels are still higher than the standard value (200 - 300 U.CARR). Thus, attention must be paid to the interpretation of each resultant index. The level of antioxidant potential of breastmilk may be higher than that of maternal antioxidant power if examined at a time closer to the early puerperium period.

With regard to the potential of breastmilk, the BAP levels in breastmilk decreased significantly during from the early puerperium up to 3 months after giving birth as postpartum days progressed. With regard to the breastmilk of women with term delivery, there are reports that activity of superoxide dismutase (SOD), i.e., one of the representative antioxidant enzymes, and concentration of polyphenol, i.e., one of the antioxidants, were significantly higher in the colostrum than in transitional milk or mature milk [23]. On the other hand, antioxidant power which was measured by the bivalent-iron reduction method was higher in colostrum than in breastmilk in the other lactational periods. The same result was obtained by DPPH radical scavenging activity [24]. High level of antioxidant potential in colostrum may alleviate the oxidative stress of newborns with an immature antioxidative defense mechanism and then contribute to the growth and development of those newborns. A study measured antioxidant potential of breastmilk (BAP levels in breastmilk) obtained from mothers who gave birth to a premature baby and found that there was an inverse correlation between 
the number of days after birth and BAP levels in breastmilk [25]. The study also pointed out that although antioxidant potential of breastmilk decreased, the average BAP levels in breastmilk 4 - 5 days after birth was higher than those in artificial milk (preterm human milk: $3807 \pm 103.5 \mu \mathrm{mol} / \mathrm{L}$ vs. artificial milk: 2671 $\pm 96.0 \mu \mathrm{mol} / \mathrm{L}$ ). In our study, we also evaluated the maternal BAP levels of term human milk and found that they were higher than those evaluated by Ezaki et al. [25] who used artificial milk. This fact has revealed that antioxidant potential of breastmilk remains higher than that of artificial milk at least until 3 months after giving birth. In addition, the BAP levels in breastmilk of women in the early puerperium were about 2.6 times higher than those in maternal plasma. Moreover, the BAP levels in breastmilk at 3 months after giving birth, which started to decrease gradually in the early puerperium period, turned out to be about 1.5 times higher than maternal BAP levels. It was pointed out that the antioxidant substance levels in breastmilk were about 2 times higher than those in maternal plasma [26], while it was reported that the average antioxidant potential of breastmilk at 3 months after giving birth was almost close to that of maternal plasma [24]. Given that antioxidant potential of breastmilk is higher in mature milk than in colostrum or transitional milk, it can be assumed that antioxidant potential is higher in breastmilk than in maternal plasma until 3 months after giving birth. The antioxidant potential of breastmilk will probably be higher than that of mother's body if it is measured at a date closer to the early puerperium. Several studies have described about specific antioxidants: concentration of vitamin $\mathrm{C}$, one of the antioxidant vitamins, is higher in breastmilk than in mother's blood [27]; lactoferrin is present in higher concentration in breastmilk, especially in colostrum, than in blood [28]; lactoferrin is most present in human body among mammals and its concentration is about 5 times higher compared with cow's milk [29].

Taken all these reports together, human breast can be considered to have an in vivo mechanism that functions to synthesize antioxidants from blood and condense them into breastmilk. Artificial milk has been improved so that its components become closer to those of breastmilk by replacing/strengthening nutrients or reducing what is redundant. Admitting that research and development of artificial milk has advanced, components of breastmilk are so complicated that much remains to be clarified. It is very meaningful to give breastmilk containing antioxidants to newborns or infants from an early stage after delivery. Therefore, it is very important to support mothers and infants so that mothers can do breast-feeding for as long a time period as possible in order to keep oxidative stress in infant's body under control.

In this study, there was no significant correlation between maternal d-ROMs levels and BAP levels in breastmilk in the 3 periods, i.e., in the early puerperium, one month after giving birth, and 3 months after giving birth. It was also revealed that maternal oxidative stress levels had nothing to do with antioxidant potential of breastmilk. There was a positive correlation between BAP levels in 
maternal plasma and those in breastmilk in the early puerperium, consequently indicating a relationship between maternal BAP levels and BAP levels in breastmilk in each period. It was also reported that when antioxidant potential was measured by using the ABTS radical method, there was a positive correlation between antioxidant components in breastmilk and the antioxidant status of mothers and newborns [26]. Moreover, a study that evaluated maternal antioxidant status and antioxidant potential of breastmilk using the FRAP method also revealed a significant correlation between them [24]. Our study, too, revealed a correlation between maternal antioxidant status and antioxidant potential of breastmilk. When maternal antioxidant potential decreases in the lactation period, maternal nutritional status should be carefully monitored and it should also be taken into consideration that the decrease might affect antioxidant potential of breastmilk and decrease the effect of antioxidant potential of breastmilk on infants.

The present study had a limitation: we were not fully able to analyze the factors that affected the oxidative stress and antioxidant power in the postpartum, as well as the antioxidant potential of breastmilk. Therefore, further study is warranted in the future to collect detailed information on maternal health and breast condition in the postpartum and the amount of breast milk in order to clarify the factors that affect each biomarker.

\section{Conclusion}

Our study successfully clarified the kinetics of oxidative stress, antioxidative power, and antioxidative potential of breastmilk of breast-feeding mothers during the period from the early puerperium up to 3 months after giving birth, as well as the relationship among these biomarkers. The d-ROMs levels in the maternal plasma decreased as the postpartum days progressed but remained at high levels even 3 months after giving birth. BAP levels were significantly low in the early puerperium and increased to the almost normal level at one month after giving birth. On the other hand, BAP levels in breastmilk were higher in the early puerperium and then gradually decreased until 3 months after giving birth. Moreover, BAP levels in breastmilk in the early puerperium were higher compared with the maternal BAP levels, and there was a positive correlation between BAP levels in breastmilk and those in the maternal plasma. When maternal antioxidant power decreases after giving childbirth, attention should be paid to maternal health status and the decrease of antioxidant potential of breastmilk. Evaluation of oxidative stress and antioxidant power can be expected to be used as the index of women's health management not only in the pregnancy and intrapartum period but also in the lactation period.

\section{Acknowledgements}

The authors thank all the participants in this study as well as the director, midwives, and other staff of each facility for their kind support for us. This study 
was carried out with the financial support of Yamaji Fumiko Nursing Research Fund.

\section{References}

[1] Halliwell, B. and Gutteridge, J.M. (2015) Free Radicals in Biology and Medicine. 5th Edition, Oxford University Press, UK. https://doi.org/10.1093/acprof:oso/9780198717478.001.0001

[2] Myatt, L. and Cui, X. (2004) Oxidative Stress in the Placenta. Histochemistry and Cell Biology, 122, 369-382. https://doi.org/10.1007/s00418-004-0677-x

[3] Hubel, C.A. (1999) Oxidative Stress in the Pathogenesis of Preeclampsia. Proceedings of the Society for Experimental Biology and Medicine, 222, 222-235. https://doi.org/10.1046/j.1525-1373.1999.d01-139.x

[4] Raijmakers, M.T., Dechend, R. and Poston, L. (2004) Oxidative Stress and Preeclampsia: Rationale for Antioxidant Clinical Trials. Hypertension, 44, 374-380. https://doi.org/10.1161/01.HYP.0000141085.98320.01

[5] Eriksson, U.J. and Borg, L.A. (1993) Diabetes and Embryonic Malformations, Role of Substrate-Induced Free-Oxygen Radical Production for Dysmorphogenesis in Cultured Rat Embryos. Diabetes, 42, 411-419. https://doi.org/10.2337/diab.42.3.411

[6] Sanaka, M., Minei, S., Suzuki, N., Yanagisawa, K. and Iwamoto, Y. (1999) Oxidative Stress and Diabetic Pregnancy. Journal of Tokyo Women's Medical University, 5, 272-279.

[7] Toescu, V., Nuttall, S.L., Martin, U., Nightingale, P., Kendall, M. J., Brydon, P., et al. (2004) Changes in Plasma Lipids and Markers of Oxidative Stress in Normal Pregnancy and Pregnancies Complicated by Diabetes. Clinical Sciences, 106, 93-98. https://doi.org/10.1042/CS20030175

[8] Schulpis, K.H., Lazaropoulou, C., Vlachos, G.D., Partsinevelos, G.A., Michalakakou, K., Gavrili, S., et al. (2007) Maternal-Neonatal 8-Hydroxy-Deoxyguanosine Serum Concentrations as an Index of DNA Oxidation in Association with the Mode of Labour and Delivery. Acta Obstetricia et Gynecologica, 86, 320-326. https://doi.org/10.1080/00016340601181706

[9] Hung, T.H., Chen, S.F., Hsieh, T.T., Lo, L.M., Li, M. J. and Yeh, Y.L. (2011) The Associations between Labor and Delivery Mode and Maternal and Placental Oxidative Stress. Reproductive Toxicology, 31, 144-150. https://doi.org/10.1016/j.reprotox.2010.11.009

[10] Dede, F.S., Guney, Y., Dede, H., Koca, C., Dilbaz, B. and Bilgihan, A. (2006) Lipid Peroxidation and Antioxidant Activity in Patients in Labor with Nonreassuring Fetal Status. European Journal Obstetrics \& Gynecology and Reproductive Biology, 124, 27-31. https://doi.org/10.1016/j.ejogrb.2005.04.014

[11] Hidaka, Y., Tada, H., Iijima, T., Yagoro, A. and Amino, N. (1995) Postgravid Health Care and Laboratory Tests. The Japanese Journal of Clinical Pathology, 11, 11011107.

[12] Kuroda, S., Watanabe, M., Santo, T., Shimizuishi, Y., Takano, T., Hidaka, Y., et al. (2010) Postpartum Increase of Serum Thioredoxin Concentrations and the Relation to CD8 Lymphocytes. Annals of Clinical Biochemistry, 47, 62-66. https://doi.org/10.1258/acb.2009.009120

[13] Alberti, A., Bolognini, L., Macciantelli, D. and Caratelli, M. (2000) The Radical Cation of N,N-Diethyl-para-Phenylendiamine, a Possible Indicator of Oxidative Stress in Biological Samples. Research on Chemical Intermediates, 26, 253-267. https://doi.org/10.1163/156856700X00769 
[14] Trotti, R., Carratelli, M., Barbieri, M., Micieli, G., Bosone, D., Rondanelli, M., et al. (2001) Oxidative Stress and a Thrombophilic Condition in Alcoholics without Severe Liver Disease. Haematologica, 86, 85-91.

[15] Dohi, K., Satoh, K., Ohtaki, H., Shioda, S., Miyake, Y., Shindo, M., et al. (2005) Elevated Plasma Levels of Bilirubin in Patients with Neurotrauma Reflect Its Pathophysiological Role in Free Radical Scavenging. In Vivo, 19, 855-860.

[16] Fujimaki,A., Watanabe,K., Mori,T., Kimura, C., Shinohara, K. and Wakatsuki, A. (2011) Placental Oxidative DNA Damage and Its Repair in Preeclamptic Women with Fetal Growth Restriction. Placenta, 32, 367-372. https://doi.org/10.1016/j.placenta.2011.02.004

[17] Kodama, H., Shinohara, H., Nukazuka, A., Narita, Y. and Yoshida M. (2008) Implications of an Oxidative Stress Marker, Serum Hydroxiperoxide Concentration, in the Medical Checkup of Pregnant Women at Around 30 Weeks of Gestation. Japanese Journal of Maternal Health, 49, 98-106.

[18] Harsem, N.K., Braekke, K. and Staff, A.C. (2006) Augmented Oxidative Stress as well as Antioxidant Capacity in Maternal Circulation in Preeclampsia. European Journal of Obstetrics Gynecology and Reproductive Biology, 128, 209-215. https://doi.org/10.1016/j.ejogrb.2005.11.014

[19] Tanaka, I. and Kitagawa, M. (2016) Changes in Oxidative Stress and Anti-Oxidative Potency during Delivery. Japanese Journal of Maternal Health, 57, 340-348.

[20] Kuramoto, N. and Kitagawa, M. (2012) Oxidative Stress and Antioxidant Capacity in the Postpartum Period. Journal of Japan Academy of Midwifery, 26, 201-210. https://doi.org/10.3418/jjam.26.201

[21] Erdem, M., Harma, M., Harma, I.M., Arikan, I. and Barut, A. (2011) Comparative Study of Oxidative Stress in Maternal Blood with that of Cord Blood and Maternal Milk. Archives of Gynecology and Obstetrics, 285, 371-375. https://doi.org/10.1007/s00404-011-1993-8

[22] Arguelles, S., Machado, M.J., Ayala, A., Machado, A. and Hervias, B. (2006) Correlation between Circulating Biomarkers of Oxidative Stress of Maternal and Umbilical Cord Blood at Birth. Free Radical Research, 40, 565-570. https://doi.org/10.1080/10715760500519834

[23] Wakana, N., Daimatsu, T., Homma, K. and Tanaka, E. (2007) Sequential Changes of the Antioxidative Enzyme Activity and Polyphenol Content in Human Milk. Journal of Japan Health Medicine Association, 16, 3-7.

[24] Zarban, A., Taheri, F., Chahkandi, T. Sharifzadeh, G. and Khorashadizadeh, M (2009) Antioxidant and Radical Scavenging Activity of Human Colostrum, Transitional and Mature Milk. Journal of Clinical Biochemistry and Nutrition, 45, 150 154. https://doi.org/10.3164/jcbn.08-233

[25] Ezaki, S., Ito, T., Suzuki, K. and Tamura, M. (2008) Association between Total Antioxidant Capacity in Breast Milk and Postnatal Age in Days in Premature Infants. Journal of Clinical Biochemistry and Nutrition, 42, 133-137. https://doi.org/10.3164/jcbn.2008019

[26] Vanderjagt, D.J., Okolo, S.N., Costanza, A., Blackwell, W. and Glew, R.H. (2001) Antioxidant Content of the milk of Nigerian Women and the Sera of Their Exclusively Breast-Fed Infants. Nutrition Research, 21, 121-128. https://doi.org/10.1016/S0271-5317(00)00261-X

[27] Salmenpera, L. (1984) Vitamin C Nutrition during Prolonged Lactation, Optimal in Infants while Marginal in Some Mothers. The American Journal Clinical Nutrition, 40, 1050-1056. 
[28] Antonsen, S., Wiggers, P., Dalhoj, J. and Blaabjerg, O. (1993) An Enzyme-Linked Immunosorbent Assay for Plasma-Lactoferrin. Concentrations in 362 Healthy, Adult Blood Donors. Scandinavian Journal of Clinical and Laboratory Investigation, 53, 133-144. https://doi.org/10.3109/00365519309088400

[29] Kiyosawa, I. (1998) Human Milk in Infant Nutrition. Kanehara \& Co., Ltd., Tokyo, 18-22.

Submit or recommend next manuscript to SCIRP and we will provide best service for you:

Accepting pre-submission inquiries through Email, Facebook, LinkedIn, Twitter, etc. A wide selection of journals (inclusive of 9 subjects, more than 200 journals) Providing 24-hour high-quality service User-friendly online submission system Fair and swift peer-review system Efficient typesetting and proofreading procedure Display of the result of downloads and visits, as well as the number of cited articles Maximum dissemination of your research work

Submit your manuscript at: http://papersubmission.scirp.org/

Or contact health@scirp.org 